\title{
Grand challenge in inflammation
}

\section{Charles A. Dinarello*}

University of Colorado at Denver, Aurora, CO, USA

*Correspondence: charles.dinarello@ucdenver.edu
Acute inflammation is a normal response to infection and is beneficial for the host to survive. However, most human diseases are due to sterile inflammation. When allowed to continue unchecked, sterile inflammation can result in autoinflammatory disorders, neurodegenerative disease, or cancer. A variety of safe and effective anti-inflammatory agents are available for short term control of acute inflammation including aspirin and non-steroidal anti-inflammatory drugs (NSAIDs). For the most part, even short term glucocorticoids are safe and effective. But attacking the upstream causes of inflammation is required for long-term treatment. There is new era of anti-inflammatory agents, which includes "biologicals" such as anti-cytokine therapies, kinase inhibitors, statins, histone deacetylase inhibitors, and PPAR agonists.

Reducing pain, inflammation, and fever with salicylate-containing plant extracts can be traced throughout written human history. One hundred fifty years ago, Felix Hoffman acetylated salicylic acid and created aspirin. Aspirin inhibits the cyclooxygenase (COX) enzymes COX-1 and COX-2, which synthesize the inflammatory mediators prostaglandins and thromboxanes. The ability to block production of prostaglandins and thromboxanes accounts for aspirin being the world's most used therapeutic agent. Second to aspirin are NSAIDs, which target COX-2 and hence the synthesis of prostaglandins, particularly PGE2. Synthetic forms of natural cortisol (termed glucocorticoids) are widely used to treat many inflammatory diseases and despite their serious side effects, glucocorticoids remain a mainstay of inflammation reduction. Yet, it is still the challenge of the pharmaceutical chemist to develop more effective and less toxic agents to treat the signs and symptoms of acute inflammation as well as the long-term consequences of chronic inflammatory diseases.

Inflammation is a dynamic process with pro-inflammatory cytokines such as tumor necrosis factor (TNF)- $\alpha$ and interleukin (IL)-1 playing central roles. A number of "biologicals" have been developed to treat inflammation by reducing the activity of specific cytokines or their receptors (anticytokine therapies), block lymphocyte trafficking into tissues, prevent the binding of monocyte-lymphocyte co-stimulatory molecules, or deplete B lymphocytes. Current anti-cytokine therapies have found a place in the treatment of autoimmune diseases, such as rheumatoid arthritis, inflammatory bowel disease, psoriasis, and others. Without question, neutralization of specific pro-inflammatory cytokines has demonstrated their causative role in inflammation and has changed the lives of millions of patients with these diseases. One drawback of anti-cytokine therapies is decreased host immune defense against infection and possibly cancer. Nevertheless, the benefits of anti-cytokine therapies outweigh the risks and the risks can be reduced. Compared to the consequences of long-term glucocorticoid treatment to stem inflammation, anti-cytokine therapies are a major improvement. Indeed, organ toxicities are rarely, if ever, observed with anti-cytokine therapies as they operate almost exclusively in extracellular rather than intracellular compartments.

Kinases that act downstream of cytokine receptors have become new targets to tame inflammation, and orally active small molecule inhibitors of intracellular signaling kinases will likely be the new frontier of antiinflammatory agents. However, because many intracellular signaling molecules are involved in normal cellular functions, the effective concentration that does not elicit organ toxicity will need to be carefully determined. Statins, a safe class of drugs used for lowering serum cholesterol, also have anti-inflammatory properties. Orally active inhibitors of histone deacetylases, which are currently in clinical use, are effective antiinflammatory drugs. Naturally occurring resolvins are also being developed as antiinflammatory agents. Thus the challenge is to develop anti-inflammatory agents that are orally active, safe, and effective for treating acute or chronic inflammation.

Received: 05 January 2012; accepted: 18 January 2012; published online: 14 February 2012.

Citation: Dinarello CA (2012) Grand challenge in inflammation. Front. Immun. 3:12. doi: 10.3389/ fimmu.2012.00012

This article was submitted to Frontiers in Inflammation, a specialty of Frontiers in Immunology.

Copyright (c) 2012 Dinarello. This is an open-access article distributed under the terms of the Creative Commons Attribution Non Commercial License, which permits noncommercial use, distribution, and reproduction in other forums, provided the original authors and source are credited. 